\title{
Learning Assessment Model in Web-Learning Based on Rough Set
}

\author{
Yan $\mathrm{Li}^{1}$ and Chen Yang ${ }^{2}$ \\ ${ }^{1}$ School of Physics \& Information Engineering, Jianghan University, Wuhan 430056, \\ P.R. China \\ junlzeng@126.com \\ ${ }^{2}$ Engineering Research Center for Information Technology on Education, Center China \\ Normal University, Wuhan 430079, P.R. China \\ ly_lj1975@sina.com
}

\begin{abstract}
Assessment is a powerful technique for improving Web-Learning achievement. In this paper we outline a learning assessment model to assessment learning effect for improving learning efficiency. This learning assessment model is based on the rough set theory. In Web-Learning process, learners' data are collected. And, the model used attribute reduction to reduce many factors that in the learning process. It found the key factors which affect the learning effect. Then, the association rules among factors have been concluded. Finally, this learning assessment model is experimented at Jianghan University.
\end{abstract}

Keywords: Learning Procedure Assessment; Rough Set; Attribute Reduction.

\section{Introduction}

Learning assessment is a powerful technique for learners to improving Web-Learning achievement. There are some methods for assessment, such as fuzzy and probability, which need accessional information. Moreover, this is difficult to get such accessional information. So, we find rough set is a better method for learning assessment. This paper constructed a learning assessment model based on rough set. The learning assessment model used rough set to assess Web-Learning. The reason is that rough set doesn't need any accessional information. It directly discovers connotative knowledge [1]. We used rough set to analyze every learner's learning characteristics in the process of Web-Learning. It had reduced many factors in the learning process. And it found the key factors which affect the learning effect. Then it concluded the association rules between factors. Finally, the paper evaluated the learners' learning process using the rule according to the learning assessment model. Therefore, learners could get personal learning assessment and improve his or her learning efficiency.

\section{Learning Assessment Framework}

\subsection{Knowledge Express}

Learning assessment is not only to assess the learning result, but also to assess all the influential factors in learning process. These factors which affect the learning effect 
can be divided into subjective factors and objective factors. This system mainly assess the learning process from the factors: learning background, learning time, learning content, learning difficulty, teaching pattern, presentation style. Followings are the concrete description.

The learning assessment model uses decision table $S=(U, C \cup\{d\}, V, f)$ to represent the knowledge system. $U=\left\{x_{1}, x_{2}, x_{3}, \ldots, x_{m}\right\}$ is composed by learning process. $x_{i}(1 \leq i \leq m)$ is the learning process of knowledge point $i . C=\left\{C_{1}, C_{2}, C_{3}, C_{4}\right.$, $\left.C_{5}, C_{6}\right\}$ is the condition attribute set. It is corresponding to \{learning background, presentation style, learning content, learning difficulty, teaching pattern, learning time $\}. d$ is the decision attribute, which represents the learning effect degree. In $V$, the value range of each condition attribute is defined as the followings: learning background $C_{l}=\{1,2,3\}=\{$ excellent, generic, poor $\}$ reflects learner's learning status; presentation style $C_{2}=\{1,2,3\}=\{$ video, text, animation $\}$; learning content $C_{3}=\{1,2$, $3\}=\{$ concept, rule, advanced rule $\}$; learning difficulty $C_{4}=\{1,2,3\}=\{$ easy, mezzo, difficult $\}$; teaching pattern $C_{5}=\{1,2,3\}=\{$ prelection pattern, drilling pattern, exploring pattern $\}$; learning time $C_{6}=\{1,2,3\}=\{$ long, mezzo, short $\}$; learning effect $\mathrm{D}=\{1,2,3\}=\{$ excellent, generic, poor $\}$.

\subsection{Learning Assessment Model}

Learning assessment model is information module, data pretreatment module, decision table, attribute reduction, rule reduction and learning assessment. Figure 1 gives the flow structure.

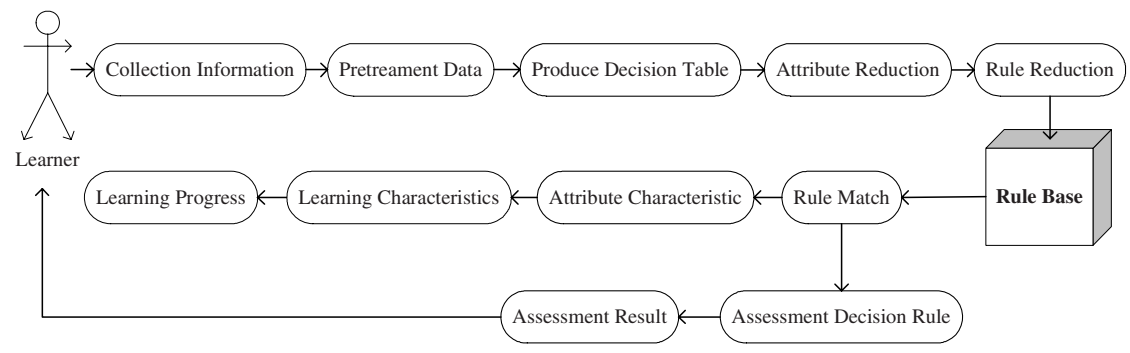

Fig. 1. Flow Structure of Learning Assessment Model

Information module mainly collects learning characteristic data, such as learning path, learning content and so on. Data pretreatment module deals with documents or other data from database. It eliminates redundant information. According to the value range of attribute, it converts consecutive and semantic data to characteristic value. Then initialization decision table. Attribute reduction is to avoid irrelevant or weak relevant attribute. And it gets the key factor which affects the learning result. Now, there are many attribute reduction methods, such as partition matrix, extendibility principle[2]. This model uses the heuristic arithmetic based on partition matrix. That makes core as start. Attribute frequency $\mathrm{p}\left(c_{\mathrm{k}}\right)$ stands the heuristic formula to reduce condition attribute. $\mathrm{p}\left(c_{\mathrm{k}}\right)$ is the times of $c_{\mathrm{k}}$ in T. Figure 2 is the arithmetic flow. 


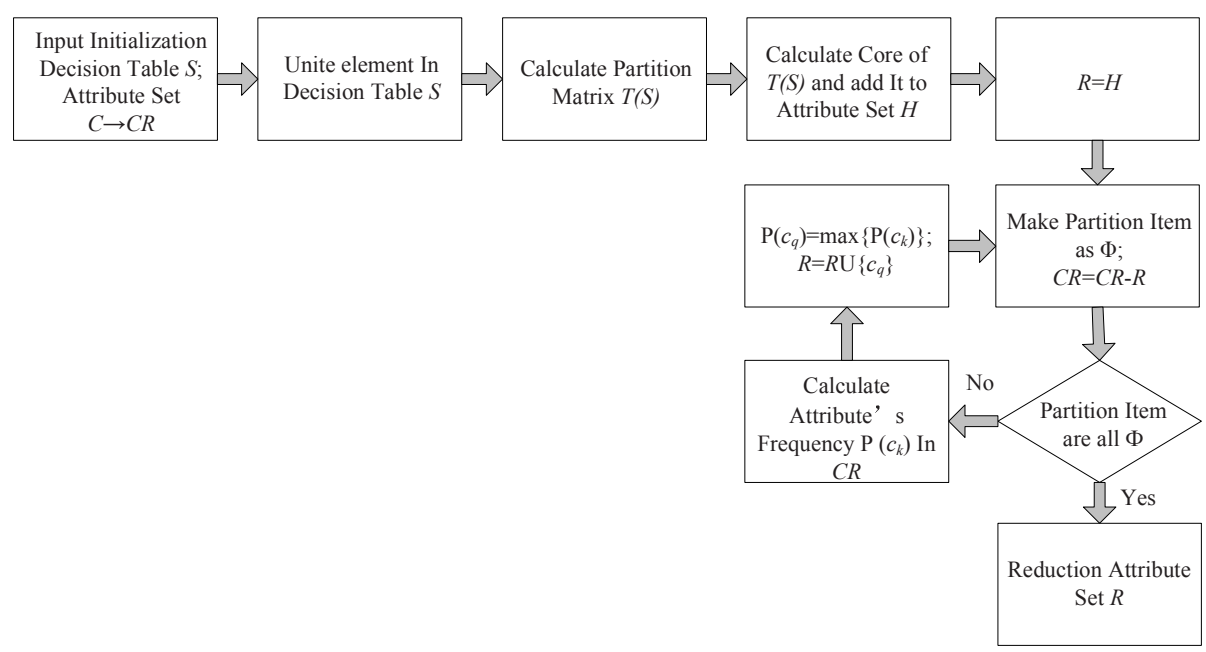

Fig. 2. Arithmetic Flow of Attribute Reduction

If there are several least attribute reduction set. The learning assessment model will have a better standard to make the best attribute reduction set. The standard is from difficult or easy degree of test and control. Condition attribute set $R$ is the key factors which affect learner's efficiency. The condition attribute set $R$ is one of the attribute assessment indexes in the assessment model.

Decision rule reduction: attribute reduction reduces condition attribute, but it is not necessary to each decision rule. Decision rule reduction uses decision making logic to eliminate the needless reduction attribute. Then a high performance decision rule is produced. These rules reflect the associated relation between learning characteristic factor and learning efficiency. This module reduces decision rule according to decision table of reduced attribute set and produces assured rules to form rule base.

Learning assessment: First it analyses attribute reduction set $R$ to help WebLearning system decide teaching tactic and teach course. The influential degree of every attribute index is confirmed by the concept of attribute importance in rough set. Secondly, learning process is assessed on the basis of rules base. The concrete method is to match between the characteristic factor and associated rules in rules base. According to matched rule, learner's learning process is evaluated. And learner can be given his or her advantage and shortcoming. Learner may be told the appropriate advice to get efficient learning. There are some situations in the rule match.

- No matched rule

- Only one matched rule

- Many matched rules, and consistent conclusion

- Many matched rules, but no consistent conclusion

If there is no matched rule, that is to say the knowledge is not enough to make a believable assessment. If there is only one matched rule or consistent conclusion, it shows that conclusion of matched rule is output firstly, then give corresponding assessment according to importance of attribute. If there is no consistent conclusion, it 
will have a synthetical assessment and select the best assessment rule. For example, $d_{1}, d_{2}, \ldots d_{r}$ are different assessment conclusions. And $r_{1}, r_{2}, \ldots r_{m}$ are the decision rules which support $d_{i}$. Then formula 1 is the assessment function of $d_{i}$.

$$
V\left(d_{i}\right)=\sum \operatorname{cov}_{j} / \sum\left(\operatorname{cov}_{j} / \operatorname{con}_{j}\right)
$$

In the above formula: $\operatorname{cov}_{j}=\left|\mathrm{X}_{i} \cap \mathrm{Y}_{j}\right| /\left|\mathrm{Y}_{j}\right|$ is the coverage of assessment rule $\mathrm{r}_{j}(1 \leq j \leq m)$, and $\operatorname{con}_{j}=\left|\mathrm{X}_{i} \cap \mathrm{Y}_{j}\right| /\left|\mathrm{X}_{i}\right|$ is the confidence of assessment rule $\mathrm{r}_{j}$. The coverage reflects the coverage degree in the equivalent decision class. The confidence reflects the probability of the trueness conclusion based on this decision rule. According to assessment function, $\mathrm{v}\left(\mathrm{d}_{1}\right), \mathrm{v}\left(\mathrm{d}_{2}\right), \ldots, \mathrm{v}\left(\mathrm{d}_{\mathrm{r}}\right)$ can be calculated. The biggest function value is selected as the best assessment for learner's learning.

This model was experimented on network teaching course of data structure in the Electronic Information Department, we found this method is welcome.

\section{Conclusion}

This paper has set up a learning assessment model on the basis of rough set. The model analyzes learning characteristics in the learning process. And the model finds association rule among factors which affect the learning efficiency. Then the model uses these rules to realize learning assessment. The model's functions are as follow: One is to clear the irrelevant or weak relevant attributes to get the key attributes. The other is finding association rule between learning characteristics and learning efficiency to get optimum teaching tactic and teaching pattern. At the end, assess the learning behavior in learning process to help Web-Learning decide next teaching activity. In the end, it improves teaching efficiency.

\section{References}

1. Pawlak, Z., Grzymalw-Busse, J., Slowinski, R., Ziarko, W.: Rough sets. Communications of the ACM. 11 (1995) 89-95

2. Keyun Hu, Yuchang LU, Chunyi SHI: Advances in rough set theory and its applications. Journal of Tsinghua University(Science and Technology). 1 (2001) 64-68

3. Greco, S., Matarazzo, B., Slowinski, R.: Rough sets methodology for sorting problems in presence of multiple attributes and criteria. European Journal of Operational Research.138 (2002) 247-259

4. Rasmussen, K. L., Davidson, G.: Hypermedia and Learning Styles: Can Performance Be Influenced. J of Education Multimedia and Hypermedia. 4 (1998) 291-308 